\title{
HFE genotypes in patients with chronic pancreatitis and pancreatic adenocarcinoma
}

Tomas Hucl, $M D^{1}$, Marja-Leena Kylanpää-Bäck, $M D^{2}$, Heiko Witt, $M D^{3}$, Beat Künzli, $M D^{4}$, Marko Lempinen, $M D^{2}$, Alexander Schneider, $M D^{1}$, Esko Kemppainen, $M D^{2}$, Matthias Löhr, $M D^{1}$, Helmut Friess, $M D^{4}$, Johann Ockenga, $M D^{5}$, Jonas Rosendahl, $M D^{6}$, Hans-Ulrich Schulz, $M D^{7}$, Thomas Gress, $M D^{8}$, Manfred V. Singer, $M D^{1}$, and Roland H. Pfützer, $M D^{1}$

\begin{abstract}
Purpose: The homozygous p.C282Y variant of the HFE gene is a major risk factor for hereditary hemochromatosis, a disorder of iron metabolism resulting in progressive iron accumulation in a variety of organs including the pancreas. Heterozygosity of p.C282Y and p.H63D may increase susceptibility to chronic liver and pancreatic disease. This study determines the frequencies of p.C282Y and p.H63D alterations in patients with chronic pancreatitis and pancreatic adenocarcinoma. Methods: In total, 958 patients (349 with alcoholic pancreatitis, 343 with idiopathic pancreatitis, 64 with familial chronic pancreatitis, 34 with acute pancreatitis, and 168 with pancreatic adenocarcinoma) were enrolled and compared with 681 healthy and 100 alcoholic controls. Furthermore, 45 parentoffspring trios were included for segregation analysis. Genotyping of p.C282Y and p.H63D was performed by restriction fragment length polymorphism or melting curve analyses. Results: No significant differences were found in heterozygosity for p.C282Y and p.H63D when patients with alcoholic (8.0/21.5\%), idiopathic (7.3/24.5\%), or familial (9.8/ 23.0\%) pancreatitis, or pancreatic adenocarcinoma (5.4/28.6\%) were compared with healthy $(6.2 / 24.8 \%)$ and alcoholic (7.0/25.0\%) controls. Neither genotype was associated with the presence of secondary diabetes mellitus in patients with chronic pancreatitis. Conclusion: Although hemochromatosis is associated with pancreatic pathology, the p.C282Y and p.H63D variants do not play a significant role in the pathogenesis of chronic pancreatitis or pancreatic adenocarcinoma. Genet Med 2007:9(7):479-483.
\end{abstract}

Key Words: hemochromatosis, HFE, genetic analysis, chronic pancreatitis, pancreatic adenocarcinoma

Chronic pancreatitis is a chronic inflammatory process of the pancreas characterized by pain and progressive fibrosis leading to exocrine and/or endocrine insufficiency. Alcoholic and idiopathic chronic pancreatitis account for the majority of cases. Patients with chronic pancreatitis are at increased risk for developing pancreatic adenocarcinoma.

Several genetic factors have been identified to predispose persons to chronic pancreatitis. Genetic alterations in the cationic trypsinogen gene (PRSS1) cause hereditary pancreatitis

From the ${ }^{1}$ Department of Medicine II, University of Heidelberg, Mannheim, Germany; ${ }^{2}$ Department of Surgery, Helsinki University Central Hospital, Helsinki, Finland; ${ }^{3}$ Department of Gastroenterology, Charité, CVK, University Medicine Berlin, Berlin, Germany; ${ }^{4}$ Department of General Surgery, University of Heidelberg, Heidelberg, Germany; ${ }^{5}$ Department of Gastroenterology, Hepatology and Endocrinology, Charité, CCM, University; Medicine Berlin, Berlin, Germany; ${ }^{6}$ Department of Medicine II, University of Leipzig, Leipzig, Germany; ${ }^{7}$ Department of Surgery, Otto-von-Guericke University, Magdeburg, Germany; and ${ }^{8}$ Department of Gastroenterology, University of Marburg, Marburg, Germany.

Disclosure: The authors declare no conflict of interest.

Roland H. Pfützer, MD, Department of Medicine II, University of Heidelberg at Mannheim, Theodor-Kutzer Ufer 1-3, 68167 Mannheim, Germany.E-mail: roland.pfuetzer@med.ma. uni-heidelberg.de.

Submitted for publication February 24, 2007.

Accepted for publication April 23, 2007.

DOI: 10.1097/GIM.0b013e3180986df4
(Online Mendelian Inheritance in Man [OMIM] 167800), and mutations in the serine protease inhibitor, Kazal type 1 (SPINK1) (OMIM 167790), and the cystic fibrosis transmembrane conductance regulator (CFTR) (OMIM 602421) genes have been associated with chronic pancreatitis. ${ }^{1-5}$ Because these alterations are present only in a subset of patients with chronic pancreatitis, other genetic determinants are likely important in predisposing humans to the disease. Homozygous and compound heterozygous alterations in the HFE gene have been reported in a family with hereditary pancreatitis and segregated with the disease. ${ }^{6}$

Hereditary hemochromatosis (HH) (OMIM 235200) is a metabolic disease characterized by accumulation of excess iron in a variety of organs, leading to their failure and the development of serious illnesses, such as cirrhosis, diabetes mellitus, cardiomyopathy, and arthritis. Homozygous p.C282Y alteration in the HFE gene is associated with iron overload (serum ferritin levels and transferrin saturation) and was reported to account for the majority of cases of HH. ${ }^{7}$ However, p.C282Y homozygosity is relatively common in the general population and by itself is insufficient to cause $\mathrm{HH} .{ }^{8} \mathrm{~A}$ second alteration (p.H63D) in a compound heterozygous (pC282Y years/ $\mathrm{pH} 63 \mathrm{D})$ or homozygous state $(\mathrm{pH} 63 \mathrm{D} / \mathrm{pH} 63 \mathrm{D})$ is associated with mild to moderate iron overload and found in a minority 
of patients with $\mathrm{HH} .{ }^{7}$ Large population-based studies recently reported slightly higher transferrin saturation in heterozygous carriers of both variants, whereas no differences were seen in serum ferritin levels. ${ }^{8} 9$ Patients with $\mathrm{HH}$ are at high risk for developing hepatocellular carcinoma, and carriers of HFE variants have been reported to bear an increased cancer risk. ${ }^{10}$

Iron has been proposed to play a significant role in the development of alcoholic liver disease by exacerbating oxidative stress-induced tissue damage. In response to iron, hepatic stellate cells produce excess collagen and other matrix proteins leading to fibrosis. ${ }^{11}$ Although similar mechanisms are crucial for the development of pancreatic fibrosis, the effect of iron on the pancreatic stellate cell has not been well studied. In a mouse model of hemochromatosis, iron overload in the pancreas resulted in fibrosis of the gland with massive macrophage infiltration and loss of secretory epithelium. ${ }^{12}$ In patients with hemochromatosis, the development of diabetes is an irrevocable step in disease progression that leads to premature death, even if excess iron is subsequently removed.

It is known that chronic excessive alcohol consumption accentuates the clinical expression of hemochromatosis. Cirrhosis is up to nine times more likely in subjects with hemochromatosis who consume more than $60 \mathrm{~g}$ of ethanol per day. ${ }^{13}$ However, no effect of HFE heterozygosity on the development and severity of alcoholic cirrhosis has been observed in most studies. ${ }^{14-16}$ Nevertheless, HFE alterations have been shown to contribute to hepatic iron accumulation ${ }^{17}$ and to severity of fibrosis in nonhereditary liver disease, such as chronic hepatitis C. ${ }^{18}$ Some investigators have shown an association of p.C282Y and nonalcoholic fatty liver disease ${ }^{19}$ and porphyria cutanea tarda. ${ }^{20}$

The aim of our investigation was to determine whether heterozygous p.C282Y and p.H63D alterations are associated with chronic pancreatitis or pancreatic adenocarcinoma.

\section{MATERIALS AND METHODS}

\section{Patients}

After ethics committee approval, informed consent was obtained and whole blood was collected from 958 consecutive patients with chronic pancreatitis, acute pancreatitis, and pancreatic adenocarcinoma. The patients were recruited at all of the participating institutions between 1997 and 2003 and originated from Germany $(n=884)$, Austria $(n=11)$, and Finland $(n=63)$. The diagnosis of chronic pancreatitis was based on previously published criteria. ${ }^{2,21}$ Patients were classified according to their diagnosis: ${ }^{2,22,23}$ alcoholic pancreatitis (349 patients: 290 male, 59 female; mean age 50 years); idiopathic pancreatitis (231 adult patients: 134 male, 97 female; mean age 49 years; and 112 children [ 50 male, 62 female; mean age 8 years]); and familial pancreatitis (64 patients, 25 male, 39 female; mean age 8 years). In addition, 34 patients with acute pancreatitis (18 male, 16 female; mean age 52 years) and 168 patients with histologically confirmed pancreatic adenocarcinoma (101 male, 67 female; average age 62 years) were included. A subset of our patients $(n=157)$ were children. For
36 children, blood for genetic analysis was available from both parents; for nine children, blood was available from one parent. Each participating institution also collected blood from randomly selected blood donors to ensure that the controls originated from the same population ( 588 of German and 93 of Finnish origin, collected between 1997 and 2003). Blood was also collected from 100 persons with chronic alcohol abuse of German origin without a history or symptoms of acute or chronic pancreatitis (recruited at Central Institute of Mental Health, Mannheim for genetic association studies). In a subset of patients $(n=135)$, hospital charts were reviewed and data regarding presence of diabetes mellitus were recorded.

\section{Mutational analysis}

Genomic DNA was isolated from anticoagulated blood by standard procedures and subjected to polymerase chain reaction (PCR) amplification using a Primus 95 Plus cycler (MWG Biotech, Ebersberg, Germany) as previously described. ${ }^{24,25}$ PCR products were subjected to enzymatic digestion using RsaI and $\mathrm{MboI}$ because p.C282Y creates an additional restriction site for RsaI and p.H63D destroys an MboI restriction site. Restriction products were separated on a 3\% NuSieve (Teknova, Hollister, $\mathrm{CA}$ ) agarose gel (RsaI) and 3\% agarose gel (MboI), and visualized by ethidium bromide staining. A subset of samples were analyzed by PCR amplification followed by a melting curve analysis using fluorescence resonance energy transfer probes and the LightCycler (Roche Diagnostics, Basel, Switzerland), as published previously with slight modifications. ${ }^{26}$

\section{Statistical analysis}

Differences between groups were compared with the $\chi^{2}$ and Fisher exact tests. Correction for multiple testing according to Bonferroni was applied where appropriate. Disease-allele association in parent-offspring trios was tested by the transmission disequilibrium test. ${ }^{27} \mathrm{~A} P$ value less than 0.05 was considered significant. Power analysis was performed (Statistical Package for the

Table 1

Demographic characteristics of the study population

\begin{tabular}{lccccc}
\hline \multicolumn{1}{c}{ Disease } & Total (\%) & Men (\%) & $\begin{array}{c}\text { Women } \\
(\%)\end{array}$ & $\begin{array}{c}\text { Mean age } \\
(\text { SD })\end{array}$ & $\begin{array}{c}\text { Age } \\
\text { range } \\
(y)\end{array}$ \\
\hline $\begin{array}{lcccccc}\text { Chronic pancreatitis } \\
\text { Alcoholic }\end{array}$ & $349(36.4)$ & $290(83.1)$ & $59(16.9)$ & $48(11)$ & $19-81$ \\
Idiopathic adults & $231(24.1)$ & $134(58.0)$ & $97(42.0)$ & $51(15)$ & $16-87$ \\
$\begin{array}{l}\text { Idiopathic children } \\
\text { Familial }\end{array}$ & $112(11.7)$ & $50(44.6)$ & $62(55.4)$ & $8(3.8)$ & $2-15$ \\
$\begin{array}{l}\text { Acute pancreatitis } \\
\text { Pancreatic } \\
\text { adenocarcinoma }\end{array}$ & $64(6.7)$ & $25(39.1)$ & $39(60.9)$ & $8(4.3)$ & $2-63$ \\
Total & $168(17.5)$ & $101(60.1)$ & $67(39.9)$ & $62(9.8)$ & $37-83$ \\
\hline
\end{tabular}

SD, standard deviation. 
Table 2

Genotype and allele frequencies of the HFE gene p.C282Y and p.H63D variants

\begin{tabular}{|c|c|c|c|c|c|c|c|c|c|c|}
\hline \multicolumn{2}{|c|}{ Genotype } & \multicolumn{7}{|c|}{ Germany } & \multicolumn{2}{|c|}{ Finland } \\
\hline p.C282Y & p.H63D & $\mathrm{ACP}$ & ICP & $\mathrm{AP}$ & FCP & PDA & $\mathrm{HC}$ & $\mathrm{AC}$ & $\mathrm{ACP}$ & $\mathrm{HC}$ \\
\hline Y/Y & $\mathrm{H} / \mathrm{H}$ & $0(0)$ & $2(0.6)$ & $0(0)$ & $0(0)$ & $1(0.6)$ & $1(0.2)$ & $0(0)$ & $0(0)$ & $0(0)$ \\
\hline $\mathrm{C} / \mathrm{Y}$ & $\mathrm{D} / \mathrm{D}$ & $0(0)$ & $0(0)$ & $0(0)$ & $0(0)$ & $0(0)$ & $0(0)$ & $0(0)$ & $0(0)$ & $0(0)$ \\
\hline $\mathrm{C} / \mathrm{Y}$ & $\mathrm{H} / \mathrm{D}$ & $7(2.4)$ & $3(0.9)$ & $0(0)$ & $0(0)$ & $2(1.2)$ & $8(1.4)$ & $1(1)$ & $1(1.6)$ & $0(0)$ \\
\hline $\mathrm{C} / \mathrm{Y}$ & $\mathrm{H} / \mathrm{H}$ & $17(5.9)$ & $22(6.4)$ & $3(8.8)$ & $6(9.4)$ & $7(4.2)$ & $30(5.1)$ & $6(6)$ & $3(4.8)$ & $4(4.3)$ \\
\hline $\mathrm{C} / \mathrm{C}$ & $\mathrm{D} / \mathrm{D}$ & $2(0.7)$ & $4(1.2)$ & $0(0)$ & $0(0)$ & $3(1.8)$ & $8(1.4)$ & $0(0)$ & $0(0)$ & $4(4.3)$ \\
\hline $\mathrm{C} / \mathrm{C}$ & $\mathrm{H} / \mathrm{D}$ & 57 (19.9) & $81(23.6)$ & $6(17.6)$ & $13(20.3)$ & $46(27.4)$ & $144(24.5)$ & $24(24)$ & $10(15.9)$ & $17(18.3)$ \\
\hline $\mathrm{C} / \mathrm{C}$ & $\mathrm{H} / \mathrm{H}$ & $203(71)$ & $231(67.3)$ & $25(73.5)$ & $45(70.3)$ & 109 (64.9) & $397(67.5)$ & $69(69)$ & $49(77.8)$ & $68(73.1)$ \\
\hline Total & & 286 & 343 & 34 & 64 & 168 & 588 & 100 & 63 & 93 \\
\hline \multicolumn{11}{|l|}{ Genotype } \\
\hline $\mathrm{C} / \mathrm{Y}$ & & $24(8.4)$ & $25(7.3)$ & $3(8.8)$ & $6(9.4)$ & $9(5.4)$ & $38(6.5)$ & $7(7)$ & $4(6.3)$ & $4(4.3)$ \\
\hline $\mathrm{H} / \mathrm{D}$ & & $64(22.4)$ & $84(24.5)$ & $6(17.6)$ & $13(20.3)$ & $48(28.6)$ & $152(25.9)$ & $25(25)$ & $11(17.5)$ & $17(18.3)$ \\
\hline$P$ value $^{a}$ & & $0.297 / 0.264$ & $0.628 / 0.645$ & $0.484 / 0.285$ & $0.378 / 0.333$ & $0.601 / 0.481$ & & & $0.715 / 0.896$ & \\
\hline \multicolumn{11}{|l|}{ Allele } \\
\hline$Y$ & & $24(4.2)$ & $29(4.2)$ & $3(4.4)$ & $6(4.7)$ & $11(3.3)$ & $40(3.4)$ & $7(3.5)$ & $4(3.2)$ & $4(2.2)$ \\
\hline$D$ & & 68 (11.9) & $92(13.4)$ & $6(8.8)$ & $13(10.2)$ & $54(16.1)$ & $168(14.3)$ & $25(12.5)$ & $11(8.7)$ & $25(13.4)$ \\
\hline$P$ value $^{a}$ & & $0.407 / 0.169$ & $0.363 / 0.599$ & $0.608 / 0.207$ & $0.454 / 0.199$ & $0.909 / 0.415$ & & & $0.719 / 0.201$ & \\
\hline
\end{tabular}

ACP, alcoholic chronic pancreatitis; ICP, idiopathic chronic pancreatitis; AP, acute pancreatitis; FCP, familial chronic pancreatitis; PDA, pancreatic ductal adenocarcinoma; HC, healthy controls; AC, alcoholic controls; C, cysteine at position 282 (wild-type); Y, tyrosine at position 282 (variant); H, histidine at position 63 (wild-type); D, aspartic acid at position 63 (variant).

${ }^{a} P$ values of each subgroup when compared with healthy controls; the first number represents the wild-type/Y genotype, and the second number represents the wild-type/Y genotype (Y allele and D allele, respectively).

Table 3

Genotype frequencies in patients with diabetes mellitus

\begin{tabular}{lcccc}
\hline \multirow{2}{*}{ Genotype } & & & \multicolumn{2}{c}{ Diabetes mellitus } \\
\cline { 5 - 5 } p.C282Y & p.H63D & & Yes (\%) & No (\%) \\
\hline Y/Y & H/H & & $0(0)$ & $0(0)$ \\
C/Y & D/D & & $0(0)$ & $3(2.9)$ \\
C/Y & H/D & & $1(3.2)$ & $2(1.9)$ \\
C/Y & H/H & & $0(0)$ & $0(0)$ \\
C/C & D/D & $1(3.2)$ & $3(2.9)$ \\
C/C & H/D & $5(16.1)$ & $20(19.2)$ \\
C/C & H/H & $24(77.4)$ & $76(73.1)$ \\
Total & & 31 & 104 \\
\hline
\end{tabular}

C, cysteine at position 282 (wild-type); Y, tyrosine at position 282 (variant); $\mathrm{H}$, histidine at position 63 (wild-type); $\mathrm{D}$, aspartic acid at position 63 (variant).

Social Sciences software; SPSS Inc., Chicago, IL) using the previously reported p.C282Y and p.H63D genotype frequencies from German and Finnish populations. ${ }^{28,29}$ The difference in frequency corresponding to odds ratios of at least 1.6 (p.C282Y) and 1.4 (p.H63D) could be detected at a power of 0.8 for the given sample sizes in patients with chronic pancreatitis and 2 and 1.7 in patients with pancreatic adenocarcinoma.

\section{RESULTS}

The demographic characteristics of the study population are summarized in Table 1. The genotype and allele distribution of the studied HFE alterations in patients and controls of German and Finnish origin are shown in Table 2. In the study population of German origin, no statistically significant difference was observed in genotype and allele frequencies between patients with alcoholic pancreatitis, idiopathic pancreatitis, familial chronic pancreatitis, acute pancreatitis, and pancreatic ductal carcinoma when compared with healthy and alcoholic controls. In the study population of Finnish origin, the distribution of genotypes and alleles was similar between patients with chronic alcoholic pancreatitis and healthy controls. Furthermore, the genotype and allele frequencies were similar and not statistically different between patients and controls of German and Finnish origin. No significant differences were detected between patients with chronic pancreatitis of different cause. Analysis of 19 informative parent-offspring trios did not reveal an increased transmission of either allele. p.H63D was transmitted in 7 of 15 families, p.C282Y in 2 of 4 trios $(P>$ $0.05)$. The presence of secondary diabetes mellitus in patients 
with chronic pancreatitis was not associated with any specific genotype or allele $(P>0.05$, Table 3$)$.

\section{DISCUSSION}

Most cases of chronic pancreatitis in industrialized countries can be attributed to alcohol abuse. However, only a subset of patients with heavy alcohol consumption develop pancreatitis. The cause of idiopathic chronic pancreatitis remains largely unknown. Some patients with a family history of pancreatitis have mutations in PRSS1 or SPINK1, while others do not. Therefore, it is hypothesized that new, yet unidentified risk factors are important in the disease development in these individuals. The discovery of genetic predispositions to chronic pancreatitis suggests that more may remain to be discovered.

The report of the p.C282Y variant segregating with the disease in a family with hereditary pancreatitis ${ }^{6}$ prompted us to investigate its role in a spectrum of sporadic and familial pancreatic diseases. To our knowledge this is the first study to focus on determining the frequency of the p.C282Y and p.H63D in patients with pancreatic disease. In the present study HFE alterations were not associated with alcoholic, idiopathic, or familial chronic pancreatitis. Furthermore, no association was seen in patients with pancreatic adenocarcinoma. In addition to healthy controls, we used patients with chronic severe alcohol abuse without chronic pancreatitis as a control group for patients with alcoholic pancreatitis. Nevertheless, comparison of patients with alcoholic pancreatitis, alcoholic patients without pancreatitis, and healthy controls did not reveal any significant differences. Our results agree with those of Frenzer et al., ${ }^{15}$ who, focusing on alcoholic cirrhosis, used a small number of patients with chronic pancreatitis as controls. In our study the observed genotypes were comparable to the previously reported data from population studies in Germany and Finland. ${ }^{28,29}$

We hypothesized that HFE variants acting through altered iron metabolism may predispose patients to fibrosis or carcinogenesis. Several possible factors may account for the lack of association between HFE genotypes and various pancreatic diseases. First, iron-related genes other than HFE may be important. Recently, other genes that play significant roles in maintaining iron homeostasis were identified..$^{30}$ Mutations in hepcidin (HAMP), iron-importer protein transferring receptor 2 (TFR2), and hemojuvelin (HJV) have been identified in patients with altered iron metabolism. ${ }^{7,30}$ Genetic alterations in these genes were not explored in our study. Second, because the HFE variants alone did not present a predisposing factor in our patients, a relationship between HFE and other genes that play a role in iron metabolism or cellular antioxidant defense may be important. The HFE wild-type gene product complexes with the transferrin receptor (TFRC). The two investigated HFE variants have been shown to increase the affinity of the receptor for transferrin and increase cellular iron uptake. Beckman et al., ${ }^{31}$ when studying multiple neoplastic disorders, found no association for HFE and TFRC genotypes separately, but an interaction between p.C282Y and p.S142G variants was found in multiple myeloma, breast, and colorectal cancer. Third, because we did not determine markers of iron metabolism in blood or tissue, we cannot completely exclude the role of iron in predisposing patients to pancreatitis or pancreatic cancer. However, even an obvious biochemical phenotype of iron overload rarely results in significant organ damage, suggesting that a simple association is unlikely. ${ }^{32}$

Because the frequency of diabetes mellitus in patients with $\mathrm{HH}$ is high, data regarding presence of secondary diabetes mellitus in our patients were recorded. However, neither alteration was a risk factor for development of diabetes mellitus in our study population.

In summary, our study provides no evidence that carriers of p.C282Y, p.H63D, or both have an increased risk for developing chronic alcoholic, idiopathic, and familial pancreatitis or pancreatic adenocarcinoma.

\section{ACKNOWLEDGMENTS}

This work was supported by a scholarship from the German Academic Exchange Service DAAD (to T. H.), SonnenfeldStiftung, Berlin (to H. W.), and DFG (Wi 2036/2-1) (to H. W.). The investigators' collaboration was established through the educational and research program "Pancreas 2000," sponsored by Solvay Pharma, Sweden. The authors thank Dr. Bugert and Dr. Neumaier (both from the University of Heidelberg, Mannheim) for providing control samples, Dr. Schumann (Central Institute of Mental Health, Mannheim) for providing alcoholic control samples, and Dr. Cunningham (Johns Hopkins University, Baltimore) for critically reading the article.

\section{References}

1. Whitcomb DC, Gorry MC, Preston RA, Furey W, et al. Hereditary pancreatitis is caused by a mutation in the cationic trypsinogen gene. Nat Genet 1996;14:141145.

2. Witt H, Luck W, Hennies HC, Classen M, et al. Mutations in the gene encoding the serine protease inhibitor, Kazal type 1 are associated with chronic pancreatitis. Nat Genet 2000;25 (2):213-216.

3. Pfützer RH, Barmada MM, Brunskill AP, Finch R, et al. SPINK1/PSTI polymorphisms act as disease modifiers in familial and idiopathic chronic pancreatitis. Gastroenterology 2000;119:615-623.

4. Sharer N, Schwarz M, Malone G, Howarth A, et al. Mutations of the cystic fibrosis gene in patients with chronic pancreatitis. N Engl J Med 1998;339 (10):645-652.

5. Cohn JA, Friedman KJ, Noone G, Howarth A, et al. Relations between mutations of the cystic fibrosis gene and idiopathic pancreatitis. N Engl J Med 1998;339 (10):653658.

6. Simon P, Zimmer KP, Domschke W, Lerch MM. Hereditary pancreatitis in a family with hemochromatosis (HFE) gene mutations. Digestion 2000;61:266.

7. Adams PC. Review article: the modern diagnosis and management of haemochromatosis. Aliment Pharmacol Ther 2006;23:1681-1691.

8. Beutler E, Felitti VJ, Koziol JA, Ho NJ, et al. Penetrance of $845 \mathrm{G} \rightarrow \mathrm{A}(\mathrm{C} 282 \mathrm{Y}) \mathrm{HFE}$ hereditary haemochromatosis mutation in the USA. Lancet 2002;359:211-218.

9. Adams PC, Reboussin DM, Barton JC, McLaren CE, et al. Hemochromatosis and iron-overload screening in a racially diverse population. N Engl J Med 2005;352: 1769-1678.

10. Dorak MT, Burnett AK, Worwood M. HFE gene mutations in susceptibility to childhood leukemia: HuGE review. Genet Med 2005;7:159-168.

11. Purohit V, Russo D, Salin M. Role of iron in alcoholic liver disease: introduction and summary of the symposium. Alcohol 2003;30:93-97.

12. Simpson RJ, Konijn AM, Lombard M, Raja KB, et al. Tissue iron loading and histopathological changes in hypotransferrinaemic mice. J Pathol 1993;171:237244. 
13. Fletcher LM, Dixon JL, Purdie DM, Powell LW, et al. Excess alcohol greatly increases the prevalence of cirrhosis in hereditary hemochromatosis. Gastroenterology 2002; 122:281-289.

14. Grove J, Daly AK, Burt AD, Guzail M, et al. Heterozygotes for HFE mutations have no increased risk of advanced alcoholic liver disease. Gut 1998;43:262-266.

15. Frenzer A, Rudzki Z, Norton ID, Butler WJ, et al. Heterozygosity of the haemochromatosis mutation, $\mathrm{C} 282 \mathrm{Y}$, does not influence susceptibility to alcoholic cirrhosis. Scand J Gastroenterol 1998;33:1324.

16. Thursz M, Mantafounis D, Yallop R. Severe alcoholic liver disease is associated with the haemochromatosis gene mutant. Gastroenterology 1997;112:1401A.

17. George DK, Goldwurm S, MacDonald GA, Cowley LL, et al. Increased hepatic iron concentration in nonalcoholic steatohepatitis is associated with increased fibrosis. Gastroenterology 1998;114:311-318.

18. Smith BC, Gorve J, Guzail MA, Day CP, et al. Heterozygosity for hereditary hemochromatosis is associated with more fibrosis in chronic hepatitis C. Hepatology 1998;27:1695-1699.

19. Bonkovsky HL, Jawaid Q, Tortorelli K, LeClair P, et al. Nonalcoholic steatohepatitis and iron: increased prevalence of mutations of the HFE gene in nonalcoholic steatohepatitis. J Hepatol 1999;31:421-429.

20. Roberts AG, Whatley SD, Morgan RR, Worwood M, et al. Increased frequency of the haemochromatosis Cys282Tyr mutation in sporadic porphyria cutanea tarda. Lancet 1997;349:321-323.

21. Sarner M, Cotton PB. Classification of pancreatitis. Gut 1984;25:756-759.

22. Ammann RW. A clinically based classification system for alcoholic chronic pancreatitis: summary of an international workshop on chronic pancreatitis. Pancreas 1997;14:215-221.

23. Etemad B, Whitcomb DC. Chronic pancreatitis: diagnosis, classification, and new genetic developments. Gastroenterology 2001;120:682-707.
24. Jouanolle AM, Fergelot P, Gandon G, Yaouanq J, et al. A candidate gene for hemochromatosis: frequency of the C282Y and H63D mutations. Hum Genet 1997;100: 544-547.

25. Jeffrey GP, Chakrabarti S, Hegele RA, Adams PC. Polymorphism in intron 4 of HFE may cause overestimation of $\mathrm{C} 282 \mathrm{Y}$ homozygote prevalence in haemachromatosis. Nat Genet 1999;22:325-326.

26. Mangasser-Stephan K, Tag C, Reiser A, Gressner AM. Rapid genotyping of hemochromatosis gene mutations on the LightCycler with fluorescent hybridization probes. Clin Chemistry 1999;45:1875-1878.

27. Terwilliger JD. A powerful likelihood method for the analysis of linkage disequilibrium between trait loci and one or more polymorphic marker loci. Am J Hum Genet 1995;56:777-787.

28. Nielsen P, Carpinteiro S, Fischer R, Cabeda JM, et al. Prevalence of the C282Y and H63D mutations in the HFE gene in patients with hereditary haemochromatosis and in control subjects from Northern Germany. Br J Haematol 1998; 103:842-845.

29. Parkkila S, Niemela O, Savolainen ER, Koistinen P. HFE mutations do not account for transfusional iron overload in patients with acute myeloid leukemia. Transfusion 2001;41:828-831.

30. Pietrangelo A, Trautwein C. Mechanisms of disease: the role of hepcidin in iron homeostasis-implications for hemochromatosis and other disorders. Nat Clin Pract Gastroenterol Hepatol 2004;1:39-45.

31. Beckman LE, Van Landeghem GF, Sikstrom C, Wahlin A, et al. Interaction between haemochromatosis and transferrin receptor genes in different neoplastic disorders. Carcinogenesis 1999;20:1231-1233.

32. Beutler E. The HFE Cys282Tyr mutation as a necessary but not sufficient cause of clinical hereditary hemochromatosis. Blood 2003;101:3347-3350.

\section{Erratum}

MacDonald DJ, Sarna L, van Servellen G, Bastani R, Giger JN, Weitzel JN. Selection of family members for communication of cancer risk and barriers to this communication before and after genetic cancer risk assessment. Genet Med 2007;9(5):275-282

Table 5 on page 279 of the above referenced article ran with an incorrect heading. The second "Pre-GCRA" column should have read "Post-GCRA." We regret the error. 